\title{
V-Shape Molecular Self-Adaption Triggered 2D Self-Assembled Polymorphism by Coadsorption of $n$-Tetradecane Solvent
}

Yujia Wang ${ }^{\ddagger}$ Jinxing $\mathrm{Li}^{\ddagger}$, Tianze Hu, Meiqiu Dong, Juntian Wu, Xinrui Miao*, Wenli Deng*

College of Materials Science and Engineering, South China University of Technology, Guangzhou 510640, People's Republic of China.

E-mail: msxrmiao@scut.edu.cn, wldeng@scut.edu.cn 


\section{Synthesis and Characterization of TPTD-Nap}

Scheme S1 for the synthesis of TPTD-Nap

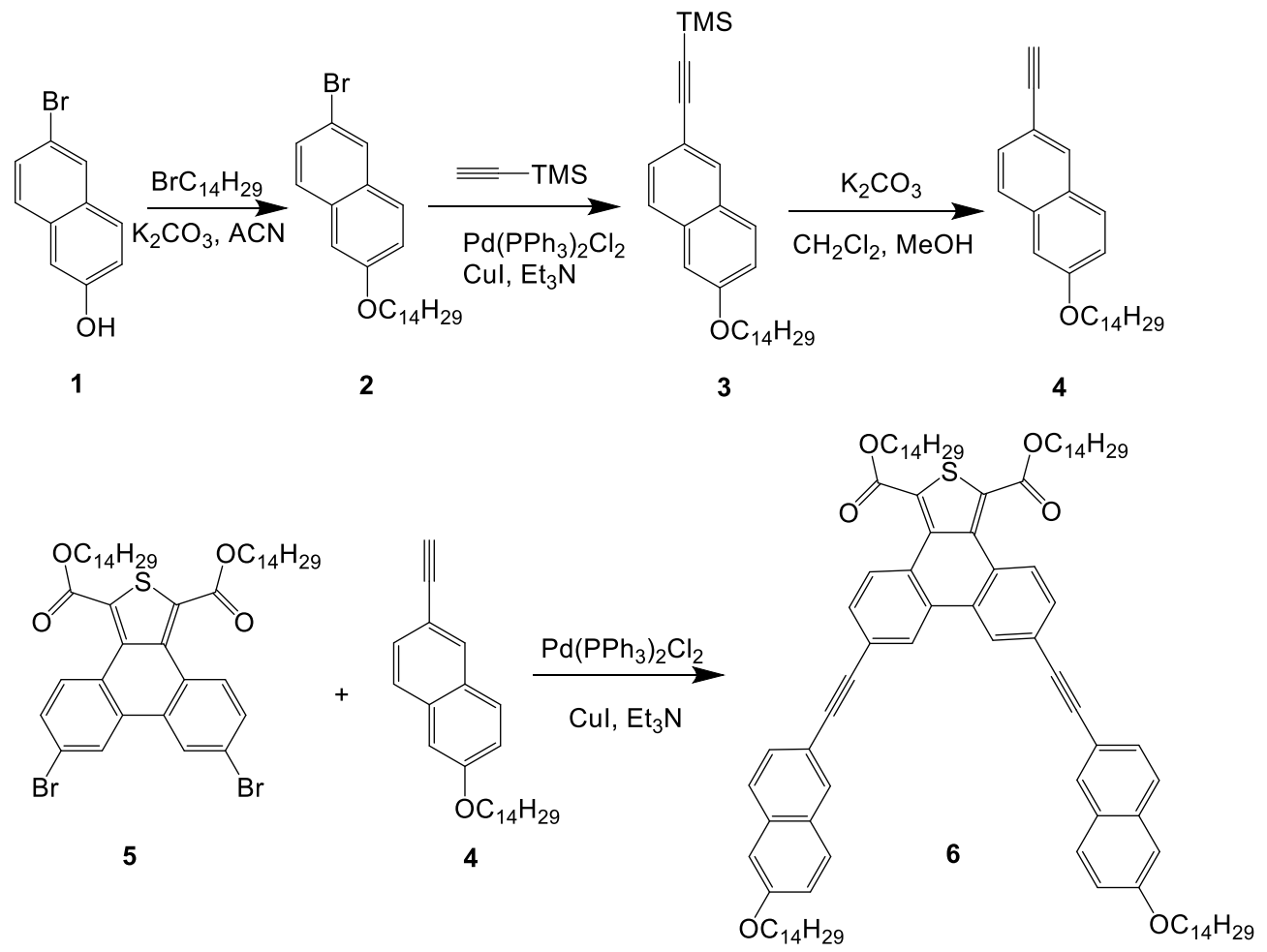

\section{2-Bromo-6-tetradecyloxy-naphthalene (compound 2)}

A mixture of 6-bromo-2-naphthol (6.00 g, $26.9 \mathrm{mmol})$, 1-bromotetradecane (13.25 g, $48 \mathrm{mmol})$, potassium carbonate $(6.60 \mathrm{~g}, 48 \mathrm{mmol})$ and acetonitrile $(100 \mathrm{ml})$, and then, this was stirred at $85{ }^{\circ} \mathrm{C}$ overnight. The solvent was removed under reduced pressure, and the obtained residue was poured into water and extracted with dichloromethane, washed with water and dried over $\mathrm{MgSO}_{4}$. After removing the solvent under reduced pressure and purifying by column chromatography eluted using hexane to yield compound 2 $(9.94 \mathrm{~g}, 88 \%)$.

${ }^{1} \mathrm{H}$ NMR (600 MHz, $\left.\mathrm{CDCl}_{3}, \mathrm{ppm}\right): \delta 7.93(1 \mathrm{H}, \mathrm{m}), 7.65(1 \mathrm{H}, \mathrm{m}), 7.60(1 \mathrm{H}, \mathrm{m}), 7.50(1 \mathrm{H}, \mathrm{m}), 7.17(1 \mathrm{H}, \mathrm{m})$, $7.11(1 \mathrm{H}, \mathrm{m}), 4.08(2 \mathrm{H}, \mathrm{m}), 1.86(2 \mathrm{H}, \mathrm{m}), 1.51(2 \mathrm{H}, \mathrm{m}), 1.28(20 \mathrm{H}, \mathrm{m}), 0.90(3 \mathrm{H}, \mathrm{m})$.

\section{2-[2-(trimethylsilyl)ethynyl]- 6-hexadecyloxy-naphthalene (compound 3)}

A mixture of 2-Bromo-6-tetradecyloxy-naphthalene (5.35 g, $12.8 \mathrm{mmol})$, trimethylsilylacetylene $(2.2$ $\mathrm{ml}, 16 \mathrm{mmol}), \mathrm{Pd}\left(\mathrm{PPh}_{3}\right)_{2} \mathrm{Cl}_{2}(0.12 \mathrm{~g}, 0.16 \mathrm{mmol})$, triethylamine $(33 \mathrm{ml})$, and $\mathrm{CuI}(0.03 \mathrm{~g}, 0.48 \mathrm{mmol})$ was stirred and at $80{ }^{\circ} \mathrm{C}$ for $24 \mathrm{~h}$. After removal of the reaction solvent at reduced pressure, petroleum ether was added $(100 \mathrm{~mL})$ and the organic layer washed with aq. $\mathrm{HC} 15 \%(50 \mathrm{~mL})$. The organic phase was dried by $\mathrm{MgSO}_{4}$, and petroleum ether was evaporated. The crude product (viscous liquid) was purified by column chromatography eluted using hexane to yield compound $3(4.75 \mathrm{~g}, 85 \%)$. 
${ }^{1} \mathrm{H}$ NMR (600 MHz, $\left.\mathrm{CDCl}_{3}, \mathrm{ppm}\right): \delta 7.74(1 \mathrm{H}, \mathrm{m}), 7.49(1 \mathrm{H}, \mathrm{m}), 7.44(1 \mathrm{H}, \mathrm{m}), 7.28(1 \mathrm{H}, \mathrm{m}), 6.96(1 \mathrm{H}, \mathrm{m})$, $6.90(1 \mathrm{H}, \mathrm{m}), 3.89(2 \mathrm{H}, \mathrm{m}), 1.67(2 \mathrm{H}, \mathrm{m}), 1.32(2 \mathrm{H}, \mathrm{m}), 1.08(20 \mathrm{H}, \mathrm{m}), 0.72(3 \mathrm{H}, \mathrm{m}), 0.10(9 \mathrm{H}, \mathrm{m})$.

\section{2-ethynyl-6-tetradecyloxy-naphthalene (compound 4)}

A mixture of 2-[2-(trimethylsilyl)ethynyl]- 6-hexadecyloxy-naphthalene (2.4 g $5.5 \mathrm{mmol})$, potassium carbonate $(3.9 \mathrm{~g}, 28 \mathrm{mmol}), \mathrm{THF}(20 \mathrm{ml})$, and $\mathrm{MeOH}(20 \mathrm{ml})$ was stirred at room temperature for $3 \mathrm{~h}$. The solvent was removed under reduced pressure, and the obtained residue was extracted with ether, washed with water and dried over $\mathrm{MgSO}_{4}$. The solvent was removed to yield compound 4 (1.38 g, 69\%).

${ }^{1} \mathrm{H}$ NMR (600 MHz, CDCl $\left.3, \mathrm{ppm}\right): \delta 7.74(1 \mathrm{H}, \mathrm{m}), 7.49(1 \mathrm{H}, \mathrm{m}), 7.44(1 \mathrm{H}, \mathrm{m}), 7.28(1 \mathrm{H}, \mathrm{m}), 6.96(1 \mathrm{H}, \mathrm{m})$, $6.90(1 \mathrm{H}, \mathrm{m}), 3.89(2 \mathrm{H}, \mathrm{m}), 1.67(2 \mathrm{H}, \mathrm{m}), 1.32(2 \mathrm{H}, \mathrm{m}), 1.08(20 \mathrm{H}, \mathrm{m}), 0.72(3 \mathrm{H}, \mathrm{m}), 0.10(9 \mathrm{H}, \mathrm{m})$.

Ditetradecyl 6,9-bis((6-(tetradecyloxy)naphthalen-2-yl)ethy nyl)phenanthro[9,10-c]thiophene-1,3 dicar-boxylate (TPTD-Nap compound 6)

A mixture of 6,9-TPTD (compound 5) ${ }^{1} 8.35 \mathrm{~g}$ (9.6 mmol), 2-ethynyl-6-hexadecyloxy-naphthalene (compound 4) $7.0 \mathrm{~g}(19.2 \mathrm{mmol}), \mathrm{Pd}\left(\mathrm{PPh}_{3}\right)_{2} \mathrm{Cl}_{2}(0.35 \mathrm{~g}, 0.48 \mathrm{mmol})$, triethylamine $(33 \mathrm{ml})$, and $\mathrm{CuI}(91 \mathrm{mg}$, $0.48 \mathrm{mmol}$ ) was stirred and at $80{ }^{\circ} \mathrm{C}$ for $24 \mathrm{~h}$. After removal of the reaction solvent at reduced pressure, petroleum ether was added $(100 \mathrm{~mL})$ and the organic layer washed with aq. $\mathrm{HCl} 5 \%(50 \mathrm{~mL})$. The organic phase was dried by $\mathrm{MgSO}_{4}$, and petroleum ether was evaporated. The crude product was purified by column chromatography eluted using hexane to yield compound $6(7.32 \mathrm{~g}, 53 \%)$.

${ }^{1} \mathrm{H}$ NMR (600 MHz, CDCl $\left.3, \mathrm{ppm}\right): \delta 8.81(2 \mathrm{H}, \mathrm{m}), 8.61(2 \mathrm{H}, \mathrm{m}), 8.01(2 \mathrm{H}, \mathrm{m}), 7.67$ (2H, m), 7.64 (2H, m), $7.62(2 \mathrm{H}, \mathrm{m}), 7.57(2 \mathrm{H}, \mathrm{m}), 7.12(2 \mathrm{H}, \mathrm{m}), 7.06(2 \mathrm{H}, \mathrm{m}), 4.39(4 \mathrm{H}, \mathrm{m}), 4.02(4 \mathrm{H}, \mathrm{m}), 1.78(8 \mathrm{H}, \mathrm{m}), 1.43(8 \mathrm{H}$, m), $1.19(80 \mathrm{H}, \mathrm{m}), 0.81(12 \mathrm{H}, \mathrm{m})$. 


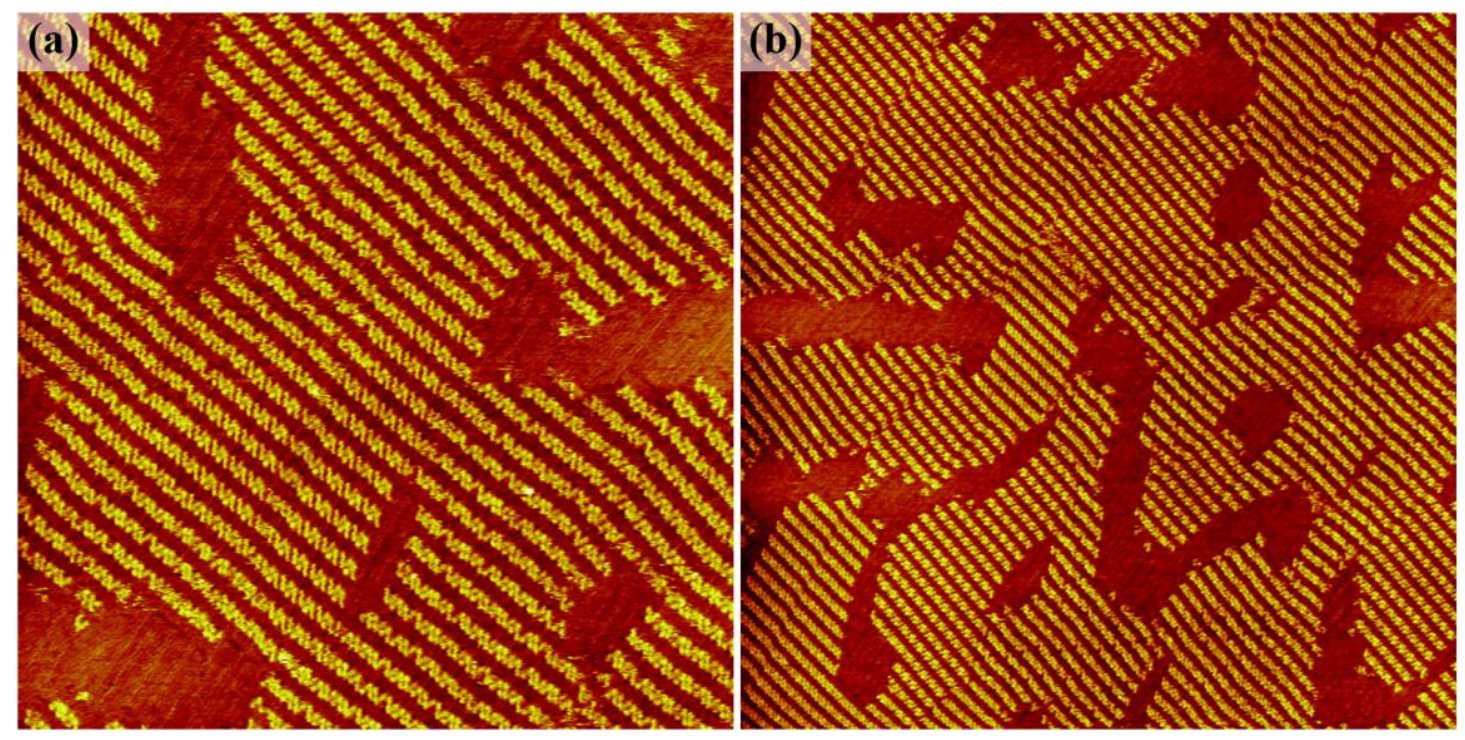

Figure S1. Large-scale STM images (a) $\left(100 \times 100 \mathrm{~nm}^{2}\right)$ and (b) $\left(200 \times 200 \mathrm{~nm}^{2}\right)$ of TPTD-Nap self-assembly at the tridecane/HOPG interface $\left(c=6.2 \times 10^{-5} \mathrm{M}\right)$. Scanning parameters: $V_{\text {bias }}=592 \mathrm{mV}$ and $I_{t}=458 \mathrm{pA}$.
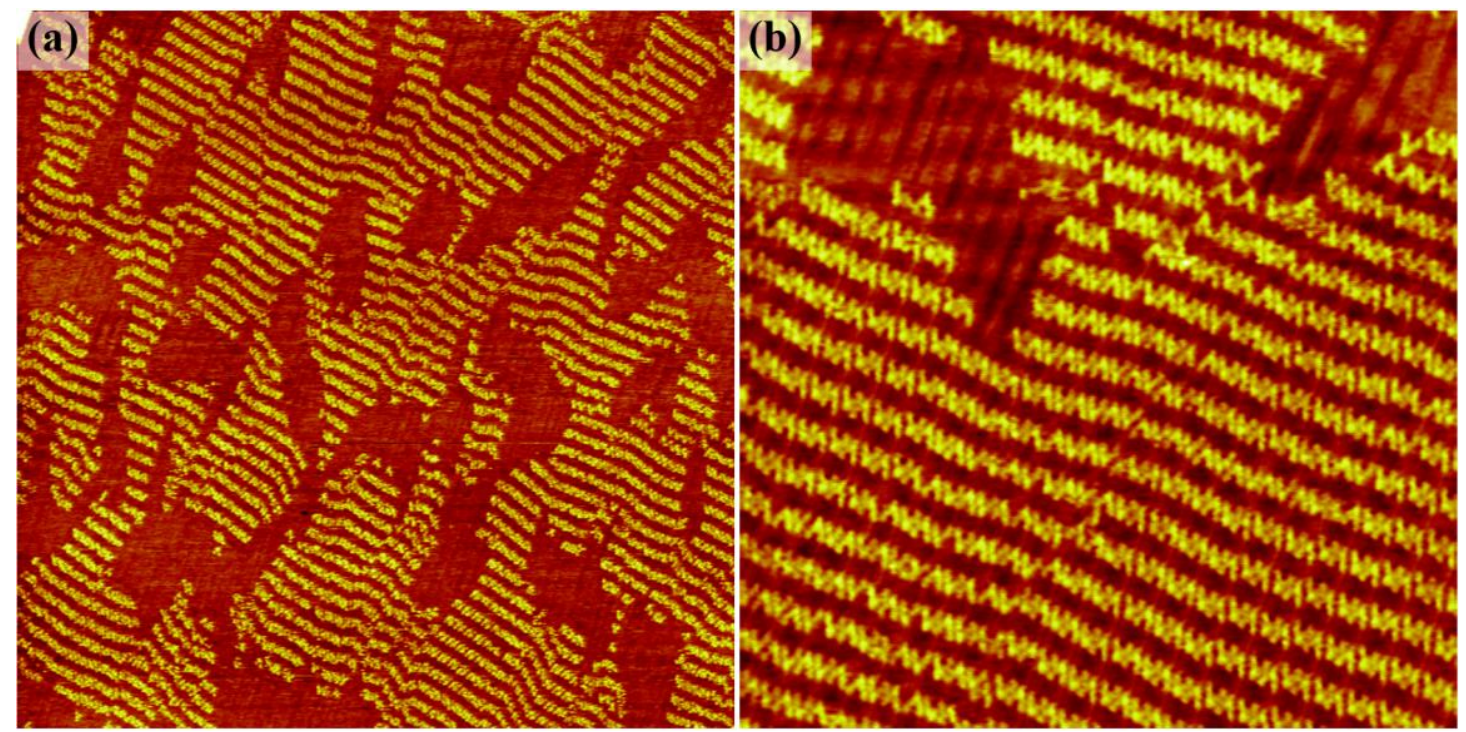

Figure S2. Large-scale STM images (a) $\left(200 \times 200 \mathrm{~nm}^{2}\right)$ and (b) $\left(60 \times 60 \mathrm{~nm}^{2}\right)$ of TPTD-Nap self-assembly at the hexadecane/HOPG interface $\left(\mathrm{c}=3.6 \times 10^{-5} \mathrm{M}\right)$. Scanning parameters: $V_{\text {bias }}=585 \mathrm{mV}$ and $I_{t}=490 \mathrm{pA}$.

\section{Reference}

1. Zha, B.; Miao, X.; Liu, P.; Wu, Y.; Deng, W. Concentration Dependent Halogen-Bond Density in the 2D Self-Assembly of a Thienophenanthrene Derivative at the Aliphatic Acid/Graphite Interface. Chem. Commun. 2014, 50, 9003-9006. 
